\title{
Polarity-Sensitive Modulation of Cortical Neurotransmitters by Transcranial Stimulation
}

\author{
Charlotte J. Stagg, ${ }^{1}$ Jonathan G. Best, ${ }^{1}$ Mary C. Stephenson, ${ }^{2}$ Jacinta O’Shea, ${ }^{1}$ Marzena Wylezinska, ${ }^{1,3}$ Z. Tamas Kincses, ${ }^{1}$ \\ Peter G. Morris, ${ }^{2}$ Paul M. Matthews, ${ }^{1,4,5}$ and Heidi Johansen-Berg ${ }^{1}$ \\ ${ }^{1}$ Centre for Functional Magnetic Resonance Imaging of the Brain, Department of Clinical Neurology, University of Oxford, Headington, Oxford OX3 9DU, \\ United Kingdom, ${ }^{2}$ Sir Peter Mansfield Magnetic Resonance Centre, University of Nottingham, Nottingham NG7 2RD, United Kingdom, ${ }^{3}$ Biological Imaging \\ Centre, Imaging Sciences Department, Medical Research Council Clinical Sciences Centre, Imperial College London, Hammersmith Campus, London W12 \\ 0NN, United Kingdom, ${ }^{4}$ Department of Clinical Neuroscience, Imperial College London, South Kensington Campus, London SW7 2AZ, United Kingdom, \\ and ${ }^{5}$ GlaxoSmithKline Clinical Imaging Centre, Hammersmith Hospital, London W12 0NN, United Kingdom
}

Transcranial direct current stimulation (tDCS) modulates cortical excitability and is being used for human studies more frequently. Here we probe the underlying neuronal mechanisms by measuring polarity-specific changes in neurotransmitter concentrations using magnetic resonance spectroscopy (MRS). MRS provides evidence that excitatory (anodal) tDCS causes locally reduced GABA while inhibitory (cathodal) stimulation causes reduced glutamatergic neuronal activity with a highly correlated reduction in GABA, presumably due to the close biochemical relationship between the two neurotransmitters.

\section{Introduction}

Transcranial direct current stimulation (tDCS) is a noninvasive stimulation technique that allows the modulation of cortical excitability in humans. Anodal tDCS leads to cortical facilitation, i.e., an overall increase in excitability, and cathodal tDCS leads to cortical inhibition (Nitsche and Paulus, 2000, 2001). The perturbation in neurophysiological measures caused by stimulation substantially outlasts the stimulation period by up to $90 \mathrm{~min}$ (Nitsche and Paulus, 2001; Nitsche et al., 2003a).

There is an increasing body of evidence from pharmacological studies suggesting possible mechanisms contributing to the associated polarity-specific modulation of cortical plasticity. Synaptic plasticity and long-term potentiation (LTP)- and long-term depression (LTD)-like changes within the neocortex depend on modulation mediated by both glutamatergic and GABAergic neurons (Trepel and Racine, 1998, 2000; Froc et al., 2000). Both the anodal facilitation and cathodal inhibition of motor-evoked potentials (MEPs) are blocked by the NMDA-receptor antagonist dextromethorphan (Liebetanz et al., 2002; Nitsche et al., 2003b). The $\mathrm{GABA}_{\mathrm{A}}$ receptor agonist lorazepam increases cortical facilitation with anodal stimulation after an initial, transient depression (Nitsche et al., 2004).

However, such pharmacological studies have inherent limitations. They do not probe local changes in isolation and primarily

Received July 18, 2008; revised Feb. 4, 2009; accepted March 13, 2009.

We thank the following for funding: Medical Research Council [C.J.S., M.W., M.C.S., Z.T.K., P.M.M., P.G.M. (including Programme Grant G9900259 to P.G.M.)], Wellcome Trust (H.J.-B.), Oxford Biomedical Research Centre (J.O'S., H.J.-B.), and the Burkitt Trust (J.G.B.). The 7 T MR Scanner in Nottingham, UK, was funded by a Joint Infrastructure Fund Grant from the Wellcome Trust.

Correspondence should be addressed to Dr. Charlotte J. Stagg, Centre for Functional Magnetic Resonance Imaging of the Brain, John Radcliffe Hospital, Headington, 0xford 0X3 9DU, UK. E-mail: cstagg@fmrib.ox.ac.uk. DOI:10.1523/JNEUROSCI.4432-08.2009

Copyright $\odot 2009$ Society for Neuroscience $\quad 0270-6474 / 09 / 295202-05 \$ 15.00 / 0$ affect modulation mediated by postsynaptic receptors. Although there is a debate as to the exact nature of the relative contributions of presynaptic and postsynaptic changes to LTP- and LTD-like phenomena, changes within the presynaptic neuron are also important (for review, see Bliss et al., 2003).

Anodal stimulation is facilitatory to the cortex. We therefore hypothesize that stimulation will decrease GABA concentration with or without a concurrent increase in glutamate. We further hypothesize that inhibitory cathodal stimulation will increase local cortical GABA concentration with or without a concurrent decrease in glutamate.

MR spectroscopy is a noninvasive imaging technique capable of measuring changes in cortical neurotransmitter concentrations from within a defined region of interest. A previous study has shown that changes in the order of $10 \%$ in the concentration of the total (MR"visible") GABA occur within the primary sensorimotor cortex in response to a motor learning paradigm in healthy controls (FloyerLea et al., 2006) suggesting that MRS is potentially sensitive to functionally relevant neurotransmitter concentration changes.

Due to the similar molecular structure of glutamate and glutamine the signals from the two cannot be resolved at $3 \mathrm{~T}$. To precisely define changes in Glx (the signal from unresolved glutamate and glutamine), a subsequent MR spectroscopy experiment was performed at $7 \mathrm{~T}$, at which field separation of the resonances from the two compounds is possible. In addition, because the GABA-optimized MRS pulse sequence by its nature precludes simultaneous acquisition of the creatine resonance, a third experiment was required to test for any concentration changes in this metabolite that might arise nonspecifically from any edema-related changes in cell volume with stimulation.

\section{Materials and Methods}

Experiment 1: neurotransmitter changes induced by tDCS at $3 \mathrm{~T}$

Subjects. Eleven healthy volunteers 11 male, mean age 27 (range 23-32 years)] participated in accordance with local ethics committee approval. 

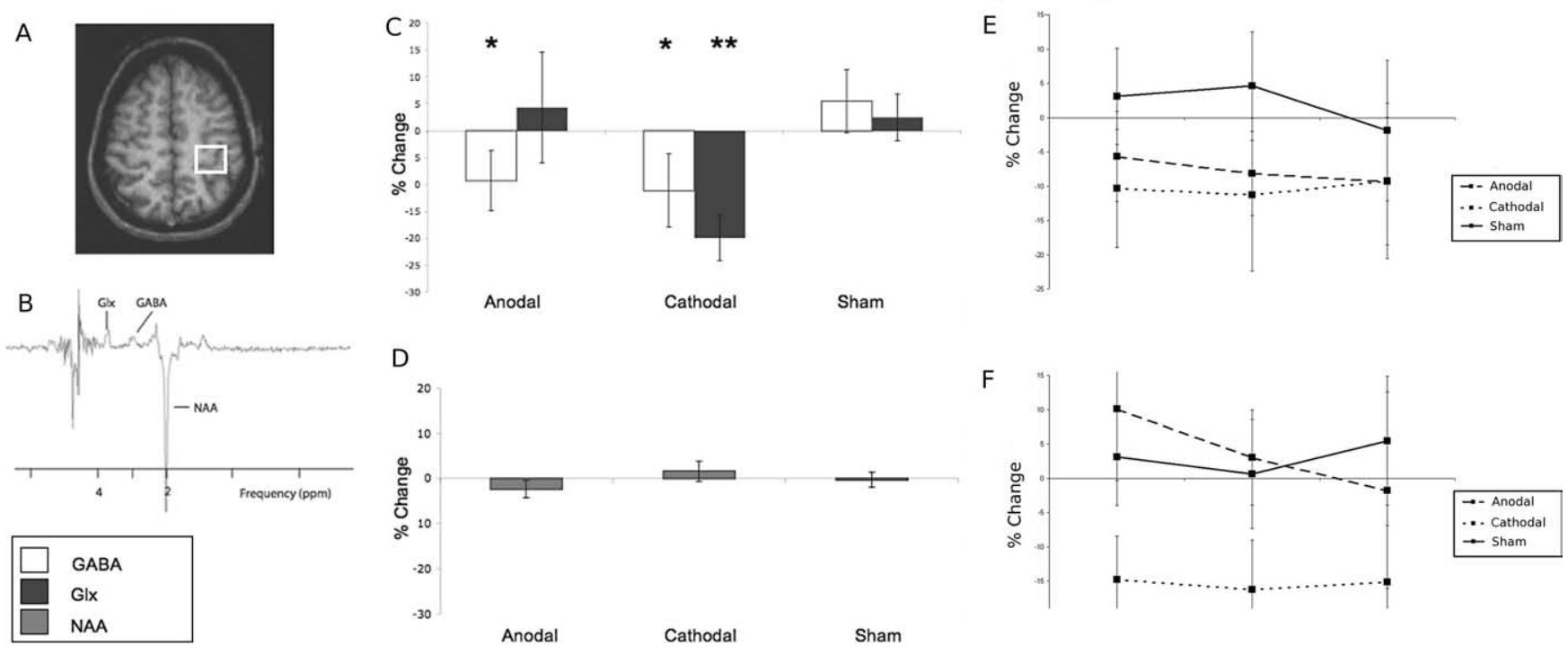

Figure 1. A, Typical placement of the $2 \times 2 \times 2 \mathrm{~cm}$ voxel within the left sensorimotor cortex. B, GABA optimized 3 T spectrum, showing resonances from NAA, GABA, and Glx. C, Changes in neurotransmitter-to-NAA ratios, given as percentage change from baseline values (mean $\pm S D$ ). ${ }^{*} p<0.05,{ }^{* *} p<0.01$. D, No change in absolute NAA quantification is seen in any stimulus condition. $\boldsymbol{E}, \boldsymbol{F}$, The decreases seen in both $\mathrm{GABA}(\boldsymbol{E})$ and $\mathrm{Glx}(\boldsymbol{F})$ following stimulation were sustained over the 20 min scanning period.

All subjects were right handed as assessed using the Edinburgh Handedness Inventory (Oldfield, 1971).

tDCS stimulation. A DC-Stimulator (Eldith) delivered a $1 \mathrm{~mA}$ current to the brain via 2 electrodes measuring $5 \times 7 \mathrm{~cm}$ (Easycap). For use in the scanner these were fitted with $5 \mathrm{k} \Omega$ resistors sited next to the electrode pad to minimize the possibility of eddy currents induced in the leads during MRS leading to heating under the electrodes. One electrode was centered on the left $\mathrm{M} 1$ and positioned $5 \mathrm{~cm}$ lateral and $2 \mathrm{~cm}$ anterior to Cz over the left hemisphere, and the other over the contralateral supraorbital ridge. High-chloride EEG electrode paste (Easycap) was used as a conducting medium between the scalp and the electrodes. For real stimulation the current had a ramp-up time of $10 \mathrm{~s}$, was held at $1 \mathrm{~mA}$ for 10 $\mathrm{min}$, and then ramped down over $10 \mathrm{~s}$. For sham stimulation the current was ramped up over $10 \mathrm{~s}$ and then immediately switched off. During MRS acquisition the electrodes were unplugged from the stimulator. For stimulation, extension leads connected the stimulator, located outside the magnetic field, to the subject within the bore of the magnet. No distortions or artifacts were seen in the resulting images as a result of the electrodes (supplemental Fig. 1, available at www.jneurosci.org as supplemental material). After stimulation was complete, leads were disconnected from the stimulator and acquisition recommenced.

Each of the 11 subjects had 3 scanning sessions, during which they received anodal, cathodal or sham tDCS in a pseudorandom order. All scanning sessions were separated by at least $48 \mathrm{~h}$ and real stimulation sessions were separated by at least 1 week. Subjects were positioned comfortably in the scanner and lay at rest for the duration of the experiment. MRS data were acquired for $\sim 15 \mathrm{~min}$ before and $20 \mathrm{~min}$ after a $10 \mathrm{~min}$ period of tDCS. No MRS data were acquired during tDCS.

MRS acquisition. A 3 T Siemens/Varian MRI system was used. Sagittal and axial T1-weighted scout images were acquired and used to place a $2 \times 2 \times 2 \mathrm{~cm}$ voxel of interest by hand over the left precentral knob, a landmark previously described to identify the hand motor representation in the human brain (Yousry et al., 1997) (Fig. 1A). To assess the creatine and NAA line widths, a standard PRESS sequence was used to acquire an unedited spectrum with 32 averages. Water was suppressed at $4.7 \mathrm{ppm}$ using a method similar to WET (Ogg et al., 1994).

The MEGA-PRESS sequence was then used to allow simultaneous spectral GABA editing, three-dimensional voxel localization, and water suppression. A selective double-banded $180^{\circ}$ pulse was created from 20 ms Gaussian pulses. The frequency of the first band of this pulse was set to $4.7 \mathrm{ppm}$ to suppress water. The second band was alternated between $1.9 \mathrm{ppm}$, the resonance frequency of $\mathrm{C} 3$ protons (strongly coupled to the observed C4 GABA protons and 3.0 ppm; condition A), and $7.5 \mathrm{ppm}$, which is symmetrically disposed about the water resonance to equalize off resonance effects (condition B). The resonance at $1.9 \mathrm{ppm}$ was inverted $180^{\circ}$ during condition $\mathrm{A}$ but not during condition $\mathrm{B}$. In condition A, the GABA C4 (triplet) resonance (at $3.0 \mathrm{ppm}$ ) therefore was fully refocused, whereas in condition $B$, this peak was not refocused, but phase modulated so that the outer triplet signals were inverted at echo time $\mathrm{TE}=68 \mathrm{~ms}$. The difference spectra from conditions $\mathrm{A}$ and $\mathrm{B}$ (at TE $=68$ $\mathrm{ms}$ ) revealed the edited GABA spectrum without the larger overlapping creatine resonance.

Three baseline sequences of GABA-optimized 96-acquisition spectra lasting $5 \mathrm{~min}$ each were performed before stimulation. Four GABAoptimized 96-acquisition sequences were acquired after stimulation.

MRS analysis. Analysis was performed using the jMRUI software package version 2.2 (http://www.mrui.uab.es/mrui). The free induction decay signal (FID) was first corrected for any nonzero DC offset and the signal was smoothed using a $2 \mathrm{~Hz}$ Lorentzian filter. The residual water signal was then filtered out by fitting and removing Gaussian peaks around the water frequency using singular value decomposition techniques. The spectrum was then phased with respect to both the zerothand first-order phase.

The nonedited PRESS acquisition was analyzed using AMARES, a nonlinear least-square fitting algorithm operating in the time domain (Vanhamme et al., 1997). Peak fitting for NAA and creatine was performed using Gaussian curves to obtain line widths for these resonances.

The GABA-optimized spectra were then analyzed using AMARES, as above. Peak fitting for the GABA and glutamate/glutamine resonances was performed using Gaussian curves with the line width constrained to that of the creatine resonance in the nonedited spectrum. The GABA and the glutamate/glutamine resonances were both fitted with 2 Gaussian peaks. The line width of the inverted NAA resonance was constrained to the line width of NAA in the unedited spectrum and a single Gaussian curve was fitted to this peak. The amplitudes of both GABA peaks were summed to give a total value for GABA, likewise summing was performed for the Glx peak.

To correct for the expected contribution from mobile brain macromolecules (MM, which include cytosolic proteins) the GABA-nulled spectrum was analyzed as above, and area under the peak resonating at 3 ppm was calculated. This was then subtracted from the GABA resonance, to give a value representing the contribution from GABA alone.

A T1-weighted structural scan was acquired in each session $(\mathrm{TR}=3 \mathrm{~s}$; $\mathrm{TE}=5 \mathrm{~ms}$, TI $=1 \mathrm{~ms}$; FOV $512 \times 256$, matrix $256 \times 128)$. FMRIB's Automated Segmentation Tool (FAST) (Smith, 2002), part of the FMRIB software library (www.fmrib.ox.ac.uk/fsl) (Smith et al., 2004) was used to 
calculate the relative quantities of gray matter and white matter within the voxel. The amplitude of the GABA and Glx peaks were corrected for the proportion of gray matter volume within the voxel [multiplied by $[\mathrm{GM}] /([\mathrm{GM}]+$ $[\mathrm{WM}]+[\mathrm{CSF}])]$ and NAA and creatine were corrected for the proportion of total brain tissue volume within the voxel [multiplied by $([\mathrm{GM}]+[\mathrm{WM}]) /([\mathrm{GM}]+[\mathrm{WM}]+[\mathrm{CSF}])]$. To allow for any changes in tissue water after stimulation, all neurotransmitter concentrations are given as a ratio to NAA, as no simultaneous creatine peak is acquired.

Analysis was conducted by two independent observers who were blind to the stimulation type used. The interobserver reliability was calculated using an interrater reliability coefficient as $\alpha=0.8801$.

\section{Experiment 2: Glu, Gln, and Cr changes at $7 \mathrm{~T}$ following} cathodal stimulation

tDCS was performed in a manner identical to that in Experiment 1, although only cathodal stimulation was used.

Subjects. Seven healthy volunteers [ 3 male; mean age 26 (range 21-33 years)] participated in accordance with local ethics committee approval. All subjects were right handed as assessed using the Edinburgh Handedness Inventory (Oldfield, 1971).

MRS acquisition and analysis. A 7 T Philips MRI system was used. Sagittal and axial T1-weighted scout images were acquired and used to place a $2 \times 2 \times 2 \mathrm{~cm}$ voxel of interest by hand over the left precentral knob as in Experiment 1. A STEAM sequence was used to acquire unedited spectra $(\mathrm{TE}=20 \mathrm{~ms}$, TM $=24 \mathrm{~ms}$, TR $=2000 \mathrm{~ms}$ ). Water was suppressed at $4.7 \mathrm{ppm}$. Four hundred forty-eight spectra were acquired before tDCS, and 608 spectra were acquired after stimulation.

Spectra were analyzed manually by a single observer blinded to the timing of the spectral acquisition. Peak amplitudes were measured by hand for each spectra independently. The amplitude of the glutamate, glutamine, and creatine peaks were recorded independently. The ratios of glutamate and glutamine to creatine were then calculated, to correct for possible changes in spectral quality over the acquisition period.

Experiment 3: Cr changes at $3 \mathrm{~T}$ following anodal stimulation tDCS was performed in a manner identical to that in Experiment 1, although only anodal stimulation was used.

Subjects. Seven healthy volunteers (4 male, mean age 24.3, range 20-49 years) participated in accordance with local ethics committee approval. All subjects were right handed as assessed using the Edinburgh Handedness Inventory (Oldfield, 1971).

MRS acquisition and analysis. A 3 T Siemens/Varian MRI system was used. The voxel of interest was placed by hand over the left precentral knob, as described in Experiment 1. A standard PRESS sequence was used to acquire unedited spectra ( $\mathrm{TR}=3 \mathrm{~s}, \mathrm{TE}=26 \mathrm{~ms}$ ). Spectra were acquired for $15 \mathrm{~min}$ before stimulation and for $20 \mathrm{~min}$ after stimulation. Water was suppressed at $4.7 \mathrm{ppm}$. MRS analysis was as for Experiment 1. Values given are absolute values of the creatine resonance. Although these do not easily equate to absolute concentration, the arbitrary units given here are reproducible within session.

\section{Results}

The neurophysiological effects of this tDCS paradigm were confirmed in a separate study (supplemental Methods, available at www.jneurosci.org as supplemental material). This demonstrated that, using these parameters, anodal stimulation led to an increase in MEP amplitude after stimulation [13 1 5\% (mean \pm $\mathrm{SE}), p=0.03$ ] and cathodal stimulation led to a decrease in MEP size $(18 \pm 7 \%, p=0.02)$.

Results from Experiment 1 showed that both GABA and Glx concentrations changed with stimulation condition [ANOVA

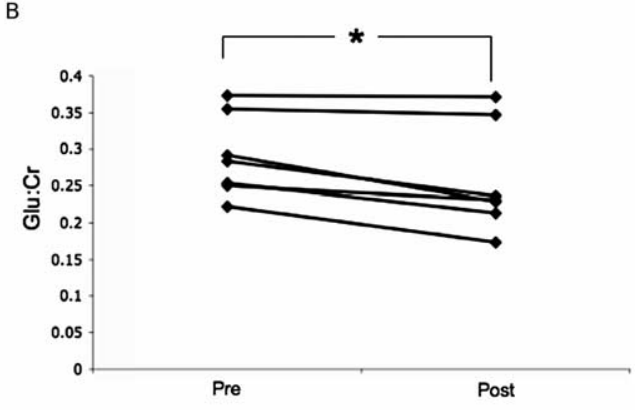

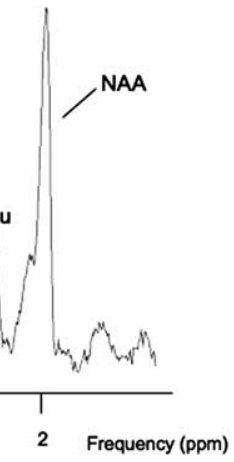

and

nces. $\boldsymbol{B}$, Glutamateum, showing creatine $(\mathrm{Cr}), \mathrm{NAA}$, glutamate $(\mathrm{Glu})$, and
sorimotor cortex in individual subjects. ${ }^{*} p<0.05$.

(main effect of stim): GABA $F_{(2,9)}=3.79, p=0.04$; Glx $F_{(2,9)}=$ 5.24, $p=0.03]$.

Following anodal stimulation, GABA concentration decreased $9.2 \pm 5.3 \%$ relative to sham [prestimulation corrected GABA:NAA ratio (mean $\pm \mathrm{SD}$ ), $0.30 \pm 0.02$; poststimulation, $0.27 \pm 0.02$; post hoc $t$ test, $p=0.03$ ]. The Glx concentration did not change (prestimulation corrected Glx:NAA ratio, $0.49 \pm$ 0.06; poststimulation, $0.49 \pm 0.07$; post hoc $t$ test, $p=0.42$ ) (Fig. $1 C)$.

Following cathodal stimulation the relative Glx concentration decreased by $19.1 \pm 4.0 \%$ compared with sham (prestimulation corrected Glx:NAA ratio, $0.51 \pm 0.09$; poststimulation, $0.39 \pm$ 0.05 ; post hoc $t$ test, $p<0.01$ ). There was also a decrease of $11.1 \pm$ $6.8 \%$ in GABA concentration (prestimulation corrected GABA: NAA ratio, $0.33 \pm 0.07$; poststimulation, $0.26 \pm 0.04$; post hoc $t$ test, $p=0.03$ ) compared with sham. There was a positive correlation between the reduction in relative GABA and Glx concentrations $\left(r^{2}=0.53, p=0.01\right)$ (Fig. 1C).

Glx includes contributions from glutamate and glutamine that cannot be separated at $3 \mathrm{~T}$. To confirm that changes measured in Glx after cathodal tDCS specifically reflect changes in glutamate concentration, we performed a separate experiment at $7 \mathrm{~T}$ (Experiment 2). This protocol enabled a clear separation of glutamate and glutamine (Fig. 2A), as well as acquisition of a simultaneous creatine $(\mathrm{Cr})$ reference peak and confirmed a $12.1 \pm 8.9 \%$ decrease in relative glutamate concentration (prestimulation Glu:Cr ratio, $0.29 \pm 0.02$; poststimulation, $0.25 \pm$ 0.02 ; $t$ test $p<0.01$ ) (Fig. $2 B$ ). There was no change in the relative glutamine concentration (prestimulation, $0.09 \pm 0.01$; poststimulation, $0.08 \pm 0.01 ; p=0.14$ ), or creatine concentration after stimulation (prestimulation Cr peak height, $5.28 \pm 0.34$; poststimulation, $4.70 \pm 0.71 ; p=0.25$ ) (Fig. $2 C$ ).

A potential confound to our interpretation of these results could arise if the stimulation induces local edema which would reduce the concentrations of all metabolites, including the reference peaks creatine and NAA. In Experiment 2 at 7 T we confirmed that there were no changes in creatine following cathodal stimulation (Fig. 2C). In Experiment 1 at $3 \mathrm{~T}$ we confirmed that there were no changes in NAA either for anodal (prestimulation, $14,115 \pm 1111$; poststimulation, $13,799 \pm 1098)$ or cathodal stimulation (prestimulation, 15,768 \pm 460; poststimulation, $16,013 \pm 548)\left(\right.$ ANOVA $\left.F_{(2,9)}=0.75, p=0.48\right)($ Fig. $1 D)$.

A simultaneous creatine peak cannot be acquired during GABA-optimized MRS. We therefore performed a further experiment (Experiment 3 ) to confirm that there were no changes in the creatine concentration after anodal stimulation at $3 \mathrm{~T}$ (pre- 
stimulation, $9.76 \pm 1.86$; poststimulation, $9.77 \pm 1.82$; $t$ test $p=$ $0.82)$.

\section{Discussion}

This study showed that facilitatory, anodal stimulation leads to a significant decrease in the GABA concentration in the cortex. In contrast, inhibitory, cathodal stimulation leads to a significant decrease in glutamate, with a correlated decrease in GABA. There was no evidence of edema occurring within the tissue as a result of the stimulation.

These results are consistent with our neurophysiological outcome measures and others (Nitsche and Paulus, 2000). Although the behavioral effects of tDCS were not investigated here, previous studies performed using similar parameters to those used here in healthy controls have been shown to exhibit behavioral modulation in a polarity specific manner; with anodal stimulation leading to an improvement in accuracy in a motor task and cathodal to a decrement (Vines et al., 2006, 2008).

\section{Anodal stimulation}

Anodal stimulation was followed by a decrease in GABA concentration. Decreases in cortical GABA over the timescale demonstrated here can be related to reduced activity of glutamic acid decarboxylase (GAD) 67 (Floyer-Lea et al., 2006). GAD-67 is the rate-limiting enzyme in the major metabolic pathway for GABA synthesis in the human cortex.

Animal models have shown that anodal stimulation increases excitatory neuronal firing rates (Kupfermann, 1965) and GAD-67 activity is reduced in conditions of increased excitatory neuronal firing (Hendry and Jones, 1988; Welker et al., 1989; Levy et al., 2002).

Evidence from previous pharmacology studies suggested that anodal stimulation might be associated with changes in glutamatergic signaling (Liebetanz et al., 2002; Nitsche et al., 2003b). However, MRS is not sensitive to modulations in NMDA receptors, only to changes in the local tissue concentration of neurotransmitters related to, e.g., altered cycling or storage. These results are not therefore directly contradictory.

\section{Cathodal stimulation}

The significant decrease in glutamate seen after cathodal stimulation is also most easily explained within the context of altered enzymatic activity in response to changes in neuronal firing. There is a high correlation between rates of cortical glucose oxidation and glutamate/glutamine cycling (Sibson et al., 1998; Rothman et al., 1999; Petroff et al., 2002). We therefore interpret the reduction in glutamate following cathodal stimulation as a consequence of a decreased rate of glutamate synthesis from glutamine with reduced excitatory neuronal transmission. This relationship has been observed in animal models, where experimentally altering the rate of neuronal activity leads to changes in neuronal-glial glutamate/glutamine cycling (Carder and Hendry, 1994).

The initially counterintuitive decrease in GABA seen after cathodal stimulation can be explained by the biochemical relations between Glx and GABA. A correlated reduction in concentrations is consistent with functional regulation of GAD-67 by the concentration of its substrate glutamate (Sonnewald et al., 1993; Martin and Tobin, 2000; Martin et al., 2000; Petroff, 2002). The lack of effect of $\mathrm{GABA}_{\mathrm{A}}$ receptor agonists on the neurophysiological changes of cathodal stimulation seen in previous studies (Nitsche et al., 2004) would further support this interpretation.

However, there must be other mechanisms involved in the effects of tDCS on the neuronal population studied here. If the effects were explained solely by a change in excitatory neuronal firing rate leading to a proportional change in glutamate production, then there would be an increase in Glx seen in response to anodal stimulation, which was not seen in our $3 \mathrm{~T}$ data. Although we did not specifically investigate the effects of anodal stimulation on glutamate at $7 \mathrm{~T}$, the sensitivity of our $3 \mathrm{~T}$ experiment excludes changes of a magnitude of $5 \%$ or greater. Although theoretically this approach might fail to detect a specific increase in glutamate, if balanced by an equal decrease in glutamine, given the close biochemical relationship of the two this would seem highly unlikely. The absence of a Glx increase following anodal stimulation is in line with the hypothesis that its primary neurophysiological effects may be driven by decreased GABAergic activity rather than increased firing rate of glutamatergic neurons but this hypothesis would need to be addressed by future studies.

\section{Possible confounds}

Any change in the volume of the local cells, caused (for example) by edema, would lead to a decrease in the levels of neurotransmitter concentrations seen here, due to the dilution effect. However, our results all are referenced to NAA. To acquire a GABAoptimized spectrum, the resonance from creatine, the standard reference resonance, is nulled. However, in a healthy control population the NAA peak should be an equally reliable reference, and certainly one that is sensitive to dilutional changes.

To definitively rule out effects of nonspecific tissue volume changes, creatine resonances were acquired in separate experiments after both anodal and cathodal stimulation. A separate experiment (Experiment 3) was required at $3 \mathrm{~T}$ to confirm no change in creatine resonance. A simultaneous creatine peak was acquired at $7 \mathrm{~T}$ during Experiment 2, and, again, no change in the creatine resonance was seen.

There are some questions raised by the current study that should be addressed in future investigations. First, due to the constraints of the technique we have only acquired data from the brain tissue in the stimulated SMC, and therefore a future study is needed to clearly distinguish the local and more general effects of tDCS on neurotransmitter levels.

In addition, we have acquired data for only $20 \mathrm{~min}$ following stimulation due to the long set up, stimulation, and scan times involved in the experiment. While we found that effects on neurotransmitters were stable over $20 \mathrm{~min}$ but it would be interesting to test their time course over longer periods. Further, our study was not powered to test for individual differences in response to stimulation due to factors such as gender. Finally, it would be interesting to determine the direct relationship between neurotransmitter changes as assessed by MRS and neurophysiological changes as assessed by TMS. However, at this point the experiments required to investigate this relationship remain technically challenging.

\section{Conclusions}

This study defines polarity-specific neurochemical changes occurring with tDCS. It seems likely that distinct local circuits are affected by the two stimulation conditions. The different neurochemical changes observed after facilitatory, anodal stimulation and after inhibitory, cathodal stimulation suggest that the excitatory effects of anodal tDCS are mediated at least in part by a reduction in GABAergic inhibition in addition to the NMDAreceptor dependency previously demonstrated (Liebetanz et al., 2002; Nitsche et al., 2003b), while the inhibitory effects of 
cathodal tDCS are mediated by reduction in excitatory glutamatergic neurotransmission.

\section{References}

Bliss T, Collingridge G, Morris R (2003) LTP: long-term potentiation; enhancing neuroscience for 30 years. Oxford: Oxford UP.

Carder RK, Hendry SH (1994) Neuronal characterization, compartmental distribution, and activity-dependent regulation of glutamate immunoreactivity in adult monkey striate cortex. J Neurosci 14:242-262.

Floyer-Lea A, Wylezinska M, Kincses T, Matthews PM (2006) Rapid modulation of GABA concentration in human sensorimotor cortex during motor learning. J Neurophysiol 95:1639-1644.

Froc DJ, Chapman CA, Trepel C, Racine RJ (2000) Long-term depression and depotentiation in the sensorimotor cortex of the freely moving rat. J Neurosci 20:438-445.

Hendry SH, Jones EG (1988) Activity-dependent regulation of GABA expression in the visual cortex of adult monkeys. Neuron 1:701-712.

Kupfermann I (1965) Effects of cortical polarization on visual discrimination. Exp Neurol 12:179-189.

Levy LM, Ziemann U, Chen R, Cohen LG (2002) Rapid modulation of GABA in sensorimotor cortex induced by acute deafferentation. Ann Neurol 52:755-761.

Liebetanz D, Nitsche MA, Tergau F, Paulus W (2002) Pharmacological approach to the mechanisms of transcranial DC-stimulation-induced aftereffects of human motor cortex excitability. Brain 125:2238-2247.

Martin DL, Tobin A (2000) Mechanisms controlling GABA synthesis and degradation in the brain. In: GABA in the nervous system: the view at fifty years (Martin DL, Olsen R, eds), pp 25-41. Philadelphia: LWW.

Martin DL, Liu H, Martin SB, Wu SJ (2000) Structural features and regulatory properties of the brain glutamate decarboxylases. Neurochem Int 37:111-119.

Nitsche MA, Paulus W (2000) Excitability changes induced in the human motor cortex by weak transcranial direct current stimulation. J Physiol 527:633-639.

Nitsche MA, Paulus W (2001) Sustained excitability elevations induced by transcranial DC motor cortex stimulation in humans. Neurology 57:1899-1901.

Nitsche MA, Liebetanz D, Antal A, Lang N, Tergau F, Paulus W (2003a) Modulation of cortical excitability by weak direct current stimulationtechnical, safety and functional aspects. Suppl Clin Neurophys 56:255-276.

Nitsche MA, Fricke K, Henschke U, Schlitterlau A, Liebetanz D, Lang N, Henning S, Tergau F, Paulus W (2003b) Pharmacological modulation of cortical excitability shifts induced by transcranial direct current stimulation in humans. J Physiol 553:293-301.

Nitsche MA, Liebetanz D, Schlitterlau A, Henschke U, Fricke K, Frommann K, Lang N, Henning S, Paulus W, Tergau F (2004) GABAergic modula- tion of DC stimulation-induced motor cortex excitability shifts in humans. Eur J Neurosci 19:2720-2726.

Ogg RJ, Kingsley PB, Taylor JS (1994) WET, a T1- and B1-insensitive watersuppression method for in vivo localized 1H NMR spectroscopy. J Magn Reson B 104:1-10.

Oldfield RC (1971) The assessment and analysis of handedness: the Edinburgh inventory. Neuropsychologia 9:97-113.

Petroff OA (2002) GABA and glutamate in the human brain. Neuroscientist 8:562-573.

Petroff OA, Errante LD, Rothman DL, Kim JH, Spencer DD (2002) Glutamate-glutamine cycling in the epileptic human hippocampus. Epilepsia 43:703-710.

Rothman DL, Sibson NR, Hyder F, Shen J, Behar KL, Shulman RG (1999) In vivo nuclear magnetic resonance spectroscopy studies of the relationship between the glutamate-glutamine neurotransmitter cycle and functional neuroenergetics. Philos Trans R Soc Lond B Biol Sci 354:1165-1177.

Sibson NR, Dhankhar A, Mason GF, Rothman DL, Behar KL, Shulman RG (1998) Stoichiometric coupling of brain glucose metabolism and glutamatergic neuronal activity. Proc Natl Acad Sci U S A 95:316-321.

Smith SM (2002) Fast, robust automated brain extraction. Hum Brain Mapp 17:143-155.

Smith SM, Jenkinson M, Woolrich MW, Beckmann CF, Behrens TEJ, Johansen-Berg H, Bannister PR, De Luca M, Drobnjak I, Flitney DE, Niazy RK, Saunders J, Vickers J, Zhang Y, De Stefano N, Brady JM, Matthews PM (2004) Advances in functional and structural MR image analysis and implementation as FSL. Neuroimage 23:S208-S219.

Sonnewald U, Westergaard N, Schousboe A, Svendsen JS, Unsgård G, Petersen SB (1993) Direct demonstration by [13C]NMR spectroscopy that glutamine from astrocytes is a precursor for GABA synthesis in neurons. Neurochem Int 22:19-29.

Trepel C, Racine RJ (1998) Long-term potentiation in the neocortex of the adult, freely moving rat. Cereb Cortex 8:719-729.

Trepel C, Racine RJ (2000) GABAergic modulation of neocortical longterm potentiation in the freely moving rat. Synapse 35:120-128.

Vanhamme L, van den Boogaart A, Van Huffel S (1997) Improved method for accurate and efficient quantification of MRS data with use of priorknowledge. J Magn Reson 129:35-43.

Vines BW, Nair DG, Schlaug G (2006) Contralateral and ipsilateral motor effects after transcranial direct current stimulation. Neuroreport 17:671-674.

Vines BW, Nair D, Schlaug G (2008) Modulating activity in the motor cortex affects performance for the two hands differently depending upon which hemisphere is stimulated. Eur J Neurosci 28:1667-1673.

Welker E, Soriano E, Van der Loos H (1989) Pliasticity in the barrel cortex of the adult mouse: effects of peripheral deprivation on GADimmunoreactivity. Exp Brain Res 74:441-452.

Yousry TA, Schmid UD, Alkadhi H, Schmidt D, Peraud A, Buettner A, Winkler P (1997) Localization of the motor hand area to a knob on the precentral gyrus. Brain 120:141-157. 